\title{
DID VERY MASSIVE STARS PRE-ENRICH AND REIONIZE THE UNIVERSE?
}

\author{
S. Peng $\mathrm{OH}^{1}{ }^{1}$ Kenneth M. Nollett ${ }^{1}$, Piero Madau ${ }^{2}$, and G.J. Wasserburg ${ }^{3}$ \\ Draft version August 12, 2013
}

\begin{abstract}
Recent studies of heavy $r$-process elements in low $[\mathrm{Fe} / \mathrm{H}]$ halo stars have suggested that an initial population of metal-free very massive stars (VMSs) may be required to provide early Fe enrichment without coproducing heavy $r$ nuclei. We find similar abundance trends in $\alpha$-elements (which should be copiously produced by VMSs), but not in other elements such as carbon (which should not), in agreement with this hypothesis. We then combine the corresponding level of prompt initial enrichment with models of VMS nucleosynthetic yields and spectra to estimate the corresponding ionizing fluxes. The result suggests that there may have been enough VMS activity to reionize the universe. The unusually hard spectrum of VMSs would imply a different reionization history from canonical models. HeII could have been reionized at high redshift, only to recombine as a subsequent generation of stars formed with a "normal" initial mass function.
\end{abstract}

Subject headings: cosmology: theory - early universe - galaxies: formation -stars: abundances

\section{INTRODUCTION}

The nature of the very first generation of metal-free ('Population III') stars has been debated for decades (Schwarzschild \& Spitzer 1953; Ezer \& Cameron 1971). It is often thought that the lack of efficient cooling mechanisms would lead to a top-heavy initial mass function (IMF) (Larson 1998), and particularly to the production of 'very massive stars' (VMSs) with $\mathrm{M}_{*}>100 \mathrm{M}_{\odot}$ (Carr, Bond \& Arnett 1984). Recent numerical simulations of cooling primordial molecular gas have hinted that this is indeed the case: they find quasistatically-contracting gas clumps with characteristic densities $\sim 10^{4} \mathrm{~cm}^{-3}$ and temperatures of a few hundred kelvin - a regime set by the properties of the chief coolant, molecular hydrogen (Bromm, Coppi, \& Larson 2001a; Abel, Bryan \& Norman 2000; Nakamura \& Umemura 2001). The Jeans unstable clumps are of order a few hundred solar masses; because the contraction is subsonic, further fragmentation is unlikely, suggesting that the very first stars were indeed very massive. Among other properties, VMSs would produce many more ionizing photons per unit stellar mass than ordinary stars (Bromm, Kudritzki, \& Loeb 2001c), and have much harder spectra; they could thus ionize HeII (Tumlinson \& Shull 2000). Their existence would have important implications for the metal enrichment and reionization history of the universe.

The duration and extent of early metal-free star formation are not known, and depend on the complex and inhomogeneous process of early metal enrichment (Gnedin \& Ostriker 1997; Madau, Ferrara, \& Rees 2001). At present, the best evidence for VMSs with $\mathrm{M}_{*}<250 \mathrm{M}_{\odot}$ may be from their distinctive nucleosynthetic products: VMSs process more of their mass into alpha-elements than normal stars, and they finally explode by a mechanism (pair instability) that is very different from core-collapse supernovae (Fryer et al. 2001), and which is unlikely to produce $r$-process elements (Heger \& Woosley 2001, here- after HW). Metal-poor halo stars provide important fossil evidence for early nucleosynthesis because they extend to metallicities even lower than those inferred for the damped Ly $\alpha$ systems (e.g., Ryan 2000).

Recent observations of $r$-process abundances as a function of $[\mathrm{Fe} / \mathrm{H}]$ in halo stars suggest that an initial population of rapidly evolving VMSs provided an intial Fe inventory without co-producing heavy $r$-nuclei (Wasserburg \& Qian 2000, hereafter WQ; Qian \& Wasserburg 2001b). In this Letter, we estimate the total number of VMSs necessary to produce the required pre-enrichment, and consider the consequences for reionization, in the spirit of previous papers (e.g. Haiman \& Loeb 1997, Miralda-Escudé \& Rees 1998) which have considered the connection between the metallicity of the $\operatorname{Ly} \alpha$ forest at $z \sim 3$ and high-redshift ionizing photon production for a 'normal' IMF. In all numerical estimates, we assume a $\Lambda$ CDM cosmology with $\left(\Omega_{M}, \Omega_{\Lambda}, \Omega_{b}, h, \sigma_{8 h^{-1}}, n\right)=$ $(0.35,0.65,0.04,0.65,0.87,0.96)$.

\section{ENRICHMENT BY VERY MASSIVE STARS}

In Figure 1 we show VMS yields as functions of mass, as computed by HW for stars in the mass range subject to pair instability explosions $M_{*} \approx 140-260 M_{\odot}$. Such stars are completely disrupted and leave no remnants. Stars with $40 \mathrm{M}_{\odot}<\mathrm{M}_{*}<100 \mathrm{M}_{\odot}$ and $M_{*}>250 \mathrm{M}_{\odot}$ are predicted instead to collapse to black holes without metal ejection (HW); thus, nucleosynthetic tracers only provide a lower limit on early star formation. The top panel shows the yields $y_{i}=M_{i} / M_{*}$ (where $M_{i}$ is the total mass of element $i$ produced, and $M_{*}$ is the initial stellar mass), assuming a He core mass of $M_{\text {core }} \approx 0.5 M_{*}$ as in HW. In zero metallicity stars the efficiency of nuclear-powered radial pulsations (Baraffe, Heger, \& Woosley 2001) and radiatively driven stellar winds (Kudritzki 2000) is greatly reduced, so mass loss is likely to be small. From the figure it is clear that iron provides an uncertain measure of VMS

\footnotetext{
${ }^{1}$ Theoretical Astrophysics, Mail Code 130-33, Caltech, Pasadena, CA 91125

${ }^{2}$ Department of Astronomy and Astrophysics, University of California Santa Cruz, Santa Cruz, CA 95064

${ }^{3}$ The Lunatic Asylum, Division of Geological and Planetary Sciences, Caltech, Pasadena, CA 91125
} 
formation. The amount of silicon burned into iron in the final VMS explosion increases with the kinetic energy of the initial collapse, and thus the iron yield varies strongly with the core mass. Yields of other nuclides depend less on $M_{*}$. The lower panel of Fig. 1 depicts the ratio of the mass of species $i$ produced in the VMS to the total mass of metals, divided by the analogous ratio for solar abundances. It illustrates the non-solar yields of VMSs. While the mass yield of oxygen is quite high, the $\alpha$ elements $\mathrm{Si}, \mathrm{S}$, and $\mathrm{Ca}$ have enrichment factors relative to solar which are far above O. These trends are specific to very low/zero-metallicity VMSs. The Si yield is about $\sim 10 \%$ in a $\sim 150 \mathrm{M}_{\odot}$ VMS which is metal-free $(\mathrm{HW})$, or has a metallicity $Z=4 \times 10^{-4} Z_{\odot}$ (Portinari et al. 1998). However, the Si yield drops by between 2 and 4 orders of magnitude for VMSs with metallicity $Z \geq 4 \times 10^{-3} Z_{\odot}$ (Table 12 of Portinari et al. 1998); likewise, the maximum Si yield of Type II supernovae ( $\mathrm{SNe}$ ) from progenitor stars of any mass and metallicity is about an order of magnitude less, $\sim 1 \%$. Thus, at low metallicities Si, S, Ca abundances should be excellent tracers of Population III VMSs. No elements above the iron group are produced in the HW models for the pair instability explosion. If these models are correct, VMSs should produce copious $\alpha$-elements with very little associated $r$-element production.

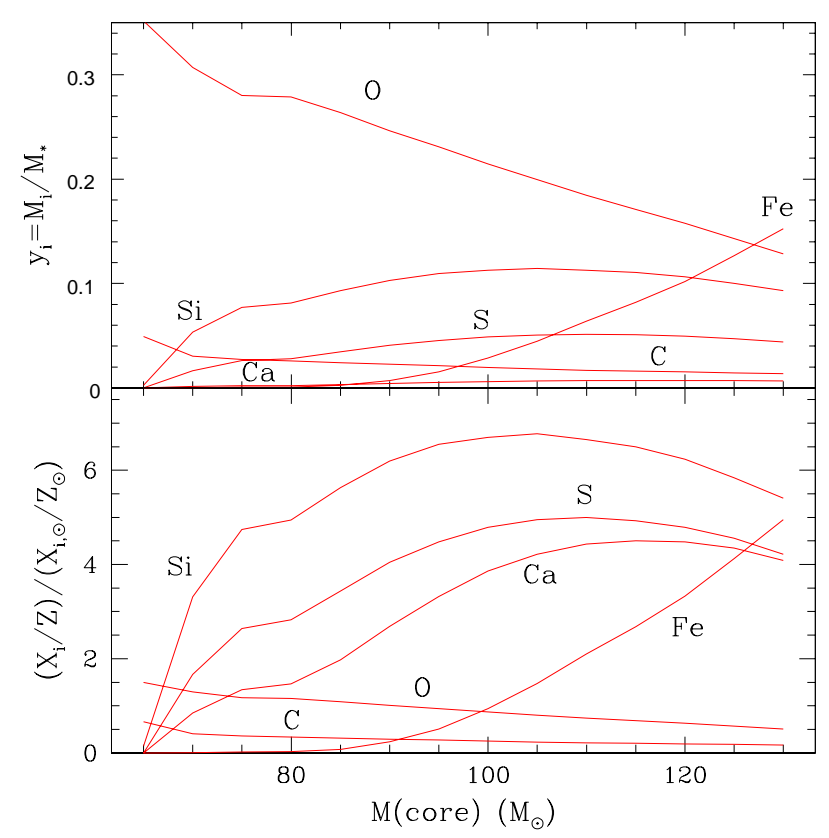

FIG. 1.- VMS yields from Heger \& Woosley (2001) used in this paper as a function of the He core mass. Top panel: the yield $y_{i}=M_{i} / M_{*}$, where $M_{i}$ is the mass of species $i$ ejected, and $M_{*}$ is the initial stellar mass [roughly twice the final He core mass M(core)]. Bottom panel: See text.

We now turn to observations. WQ analysed data from observations of heavy $r$-process abundances in metal-poor halo stars as a function of $[\mathrm{Fe} / \mathrm{H}]$ (see Fig. 2, top left). They found the following trend in the data. At the lowest metallicities, $[\mathrm{Fe} / \mathrm{H}]<-3$, the $\mathrm{Ba}$ abundance is roughly constant. Then, over a small range in $\mathrm{Fe}$ abundance, $-3.1 \leq[\mathrm{Fe} / \mathrm{H}] \leq 2.5$, there is a wide spread in the $\mathrm{Ba}$ abundance of order 2 dex. They interpreted this sudden increase in the $\mathrm{Ba}$ abundance dispersion as follows. Previous studies of radioactive ${ }^{129} \mathrm{I}$ and ${ }^{182} \mathrm{Hf}$ in meteorites appear to require two distinct classes of Type II SNe to account for $r$-process elemental abundances: high-frequency events (H) with characteristic timescales $\sim 10^{7}$ yrs that produce heavy $r$-process elements with mass numbers $A>130$ (e.g. $\mathrm{Ba}, \mathrm{Eu})$ and low-frequency events $(\mathrm{L})$ with characteristic timescales $\sim 10^{8}$ yrs that produce light $r$-process elements with mass numbers $A<130$ (e.g. iodine) (Wasserburg, Busso, \& Gallino 1996). Because of their shorter lifetime, the $\mathrm{H}$ events are the first $\mathrm{SNe}$ to explode. As they are not associated with significant coproduction of iron, they will produce a wide scatter in $\mathrm{Ba}$ abundance over a small range in $[\mathrm{Fe} / \mathrm{H}]$. However, the region $[\mathrm{Fe} / \mathrm{H}]<-3$, where the Ba abundance is very low and roughly constant, was considered by WQ and Qian \& Wasserburg (2001b) to be an initial or prompt inventory dominated by the contributions of very low-metallicity massive first-generation stars, which appear before $\mathrm{H}$ events and produce very small amounts of $r$-process elements relative to their iron production rate. In this case, the iron enrichment up to $[\mathrm{Fe} / \mathrm{H}] \sim-3$ is almost exclusively due to VMSs. The finite $\mathrm{Ba}$ abundance at $[\mathrm{Fe} / \mathrm{H}]<-3$ can be associated with very minor coproduction of $\mathrm{Ba}$ in VMSs, or trace contamination by $\mathrm{H}$ events (see WQ and Qian \& Wasserburg 2001b).
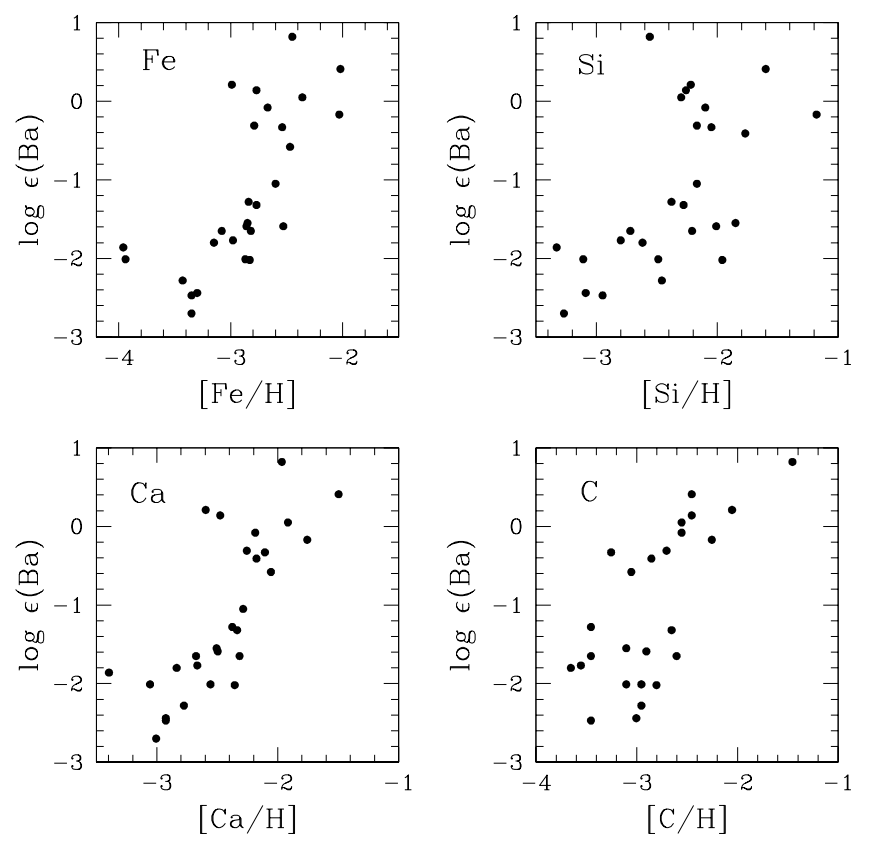

FIG. 2.- Observed Ba abundances vs. other metal abundances in metal poor halo stars, from the data of McWilliam et al. (1995). The original trend found by Wasserburg \& Qian (2000) was in Ba vs. $[\mathrm{Fe} / \mathrm{H}]$; the sharp increase in scatter of $\mathrm{Ba} / \mathrm{H}$ at fixed $[\mathrm{Fe} / \mathrm{H}] \sim-3$ also occurs in elements overproduced by VMSs, such as $\mathrm{Si}$ and $\mathrm{Ca}$, suggesting that the sources of $\mathrm{Ba}$ and $\mathrm{Fe}, \mathrm{Si}$, Ca elements are distinct. Elements not strongly overproduced by VMSs, such as C, do not show such a trend.

From our previous discussion we would expect the trend seen in Fe to be most strongly exhibited in the $\alpha$ elements. In Fig.2, we plot halo-star $\mathrm{Ba} / \mathrm{H}$ values against $\alpha$ element abundances, using the data of McWilliam et al. (1995). Indeed, $\mathrm{Si}$ and $\mathrm{Ca}$ show the same trend of a low Ba abundance at very low metallicity followed by a wide scatter over a small range in metallicity. On the other hand, we would expect these trends to be absent in elements less 
abundantly produced by VMSs, which do not experience significant initial enrichment before normal star formation takes over. This is indeed seen: carbon shows merely a linear correlation between $\mathrm{C}$ and $\mathrm{Ba}$ production with a great deal of scatter (lower right panel). This provides additional supporting evidence for early VMS formation. Most importantly, while VMS Fe yields vary strongly as a function of mass, $\mathrm{Si}$ and $\mathrm{Ca}$ yields do not change widely above $M_{\text {core }}>70 \mathrm{M}_{\odot}\left(M_{*}>150 \mathrm{M}_{\odot}\right.$, from from eqn. 1 of HW). In $\S 3$ we will use these yields to quantify the amount of early VMS formation.

Although oxygen is the element produced most abundantly in VMSs, unfortunately we cannot use it to measure VMS formation. Oxygen abundance estimates in low metallicity stars are uncertain, with different trends of $[\mathrm{O} / \mathrm{Fe}]$ vs. $[\mathrm{Fe} / \mathrm{H}]$ claimed by different observers (e.g., Israelian et al. 2001; Carretta et al. 2000). Moreover, to the best of our knowledge, there is no substantial body of simultaneous $\mathrm{O}$ and $r$-process abundances in halo stars. Note that the predicted $\mathrm{O}$ yields of VMSs are similar to SNII yields, and so the nucleosynthetic signature of VMSs may be less distinct in $\mathrm{O}$ than in the $\alpha$ elements (see, however, Qian \& Wasserburg 2001a).

\section{CAN VERY MASSIVE STARS REIONIZE THE UNIVERSE?}

VMSs are $\sim 1-2$ orders of magnitude more efficient than normal stars with a standard IMF at producing ionizing photons, per unit stellar mass (Bromm et al 2001c). Thus, even if the cooling and star formation efficiencies in gas with primordial composition are low, a much smaller fraction of the gas needs to be turned into stars in order to reionize the universe. We now attempt to determine if VMSs could indeed have reionized the universe. The onset of the large increase in $\log \epsilon(\mathrm{Ba})$ occurs when the abundances of $\mathrm{Si}$ and $\mathrm{Ca}$ are $[\mathrm{Si} / \mathrm{H}]_{\text {jump }} \approx-2.3$ and $[\mathrm{Ca} / \mathrm{H}]_{\text {jump }} \approx-2.4$. Let us assume that the IGM was uniformly polluted by VMSs up to this critical metallicity. ( $\mathrm{Si}$ abundances in the $\mathrm{Ly}-\alpha$ forest yield $[\mathrm{Si} / \mathrm{H}] \sim-2.3$, with a scatter of a factor $\sim 3$ [Cowie \& Songalia, 1998; Ellison et al., 2000], in good agreement with our estimate of $[\mathrm{Si} / \mathrm{H}]_{\text {jump. }}$.) The number density $n_{i}$ of species $i$ relative to hydrogen $\left(n_{i} / n_{H}\right)$ at the critical metallicity is determined by the fraction $f_{\mathrm{VMS}}$ of baryonic matter that was processed in VMSs. Using $[i / H]_{\text {jump }}=\log \left(\frac{\left(n_{i} / n_{H}\right)_{\text {jump }}}{\left(n_{i} / n_{H}\right) \odot}\right)$, we have

$f_{\mathrm{VMS}}=\left(n_{i} / n_{H}\right)_{\mathrm{jump}} /\left(n_{i} / n_{H}\right)_{\mathrm{VMS}}=X_{i, \odot} \times 10^{[i / \mathrm{H}]_{\mathrm{jump}}} / y_{i}$.

Here, $X_{i, \odot}$ is the mass fraction of species $i$ in the sun and $y_{i}$ is the mass yield of species $i$ from a VMS. From Fig. 1, we have $y_{\mathrm{Si}}=0.05-0.1$ for all the stars with $150 M_{\odot}<M_{*}<250 M_{\odot}$. We thus estimate that a fraction $f_{\mathrm{VMS}} \sim 3-7 \times 10^{-5}$ of all baryons must have been processed through VMSs. Ca yields a similar range, but extending up to $1.2 \times 10^{-4}$. The result is fairly insensitive to the assumed IMF since $\mathrm{Ca}$ and in particular Si yields are fairly constant for $M_{\text {core }}>70 M_{\odot}\left(M_{*}>150 M_{\odot}\right)$. An IMF dominated by VMSs with $M_{*}<150 M_{\odot}$ can be excluded since these stars produce very little $\mathrm{Fe}$, and the observed jump in $\mathrm{Ba}$ abundance at $[\mathrm{Fe} / \mathrm{H}] \sim-3$ would not be seen.

In the above estimate we assume that metals produced by VMSs are uniformly mixed throughout the IGM, and this is a fairly uncertain assumption. It is possible that early star formation was highly biased and VMSs only polluted some small fraction of the IGM from which the halo stars formed. Our estimate of $f_{\mathrm{VMS}}$ would then be correspondingly reduced. Nonetheless, metals have been detected even in underdense gas in the IGM at $2<z<3$, far from star forming regions (Schaye et al 2000), plausibly the result of early uniform enrichment by PopIII stars. We can use extended Press-Schechter theory to compute the extent to which VMS debris should be over-represented in the Galactic halo; for typical assumptions, the enhancement factor is only $\sim 1.6$ (Madau \& Rees 2001).

How many ionizing photons does our estimated $f_{\mathrm{VMS}}$ correspond to? Bromm et al (2001c) have computed models of VMS spectra and find that for $300 \mathrm{M}_{\odot}<\mathrm{M}_{*}<$ $1000 \mathrm{M}_{\odot}$ VMSs, the stellar effective temperature and luminosity per solar mass are approximately constant. From their models, a fraction $f_{* \text {, BKL }} \sim(10,1,4) \times 10^{-5}$ of the baryons in the universe must have been processed through VMSs to obtain 10 (HI,HeI,HeII) ionizing photons per $\mathrm{H}$ and He nucleus in the universe respectively (see their Table 1 and eq. 9). From this, we estimate that VMSs must have radiated $f_{\text {photon }} \sim 10 f_{\mathrm{VMS}} / f_{* \text { BKL }} \sim(3-7,30-70,8-$ 15) photons above the (HI,HeI,HeII) ionization threshold, per $\mathrm{H}$ or He nucleus. This is probably an overestimate, because the stars which eject metals have lower masses, $150-250 \mathrm{M}_{\odot}$, than the stars considered by Bromm et al. (2001b). For $100 \mathrm{M}_{\odot}$ stars the number of ionizing photons per solar mass is reduced by a factor $\sim 1.5-2$ for $\mathrm{HI}$ and HeI and $\sim 4$ for HeII (Bromm et al 2001c; Tumlinson \& Shull 2000). The uncertainties in our estimate are almost certain to be dominated by our assumption of uniform metal mixing, and by our neglect of more massive stars $M_{*}>260 \mathrm{M}_{\odot}$ which do not eject metals but collapse to black holes.

Could these ionizing photons escape from their host halos? Suppose that VMSs originated in the first pregalactic objects able to cool within a Hubble time, i.e. in halos of mass $\mathrm{M}>\mathrm{M}_{\text {crit }}=4 \times 10^{5}\left(\frac{1+\mathrm{z}}{25}\right)^{-2} \mathrm{M}_{\odot}$, (Fuller \& Couchman 2000; Haiman, Thoul \& Loeb 1996). Gas within such small halos is quickly ionized by the flux from a single VMS, and the escape fraction of ionizing photons is therefore likely to be of order unity. We obtain the density profile of the gas $n(r)$ by assuming it is initially isothermal at the virial temperature and demanding hydrostatic equilibrium within an NFW dark matter profile (Makino et al 1998). The maximum recombination rate is $\dot{N}_{\text {rec }} \approx \alpha_{B} \int n^{2}(r) 4 \pi r^{2} d r=3 \times$ $10^{48}\left(\frac{\sigma_{v}}{5 \mathrm{~km} \mathrm{~s}^{-1}}\right)^{3}\left(\frac{1+z}{25}\right)^{3 / 2} \mathrm{~s}^{-1}$. This is much smaller than the VMS HI ionizing photon production rate, $\dot{N}_{\text {ion }} \approx$ $5 \times 10^{50}\left(\frac{M_{*}}{300 M_{\odot}}\right)$ photons $^{-1}$, and so the halo gas remains wholly ionized. Moreover, gas within these small halos is easily photoevaporated: the baryonic gravitational binding energy, $E_{b} \sim 7 \times 10^{48}\left(\frac{M}{5 \times 10^{5} M_{\odot}}\right)^{5 / 3}[(1+z) / 20]$ $\mathrm{erg}$, is considerably less than the total ionizing radiation produced by a VMS, $E_{\text {ion }} \sim 10^{54}\left(M_{*} / 200 \mathrm{M}_{\odot}\right) \mathrm{erg}$. The entire baryonic component of the halo will be photoevaporated on the sound-crossing timescale of the halo, $t_{\text {evap }} \sim \frac{1}{3} r_{\text {vir }} / c_{s} \sim 3 \times 10^{6}\left(\frac{\sigma_{v}}{5 \mathrm{kms}^{-1}}\right)\left(\frac{1+z}{25}\right)^{-3 / 2}$ yrs, comparable to the lifetime of the VMS, $t_{*} \sim 3 \times 10^{6} \mathrm{yr}$. 
The amount of reionization is determined by competition between ionization and recombination. The increased mean density of the IGM at high redshift $n \propto(1+z)^{3}$ significantly increases recombination rates. On the other hand, the gas clumping factor due to dense non-linear objects $C \equiv\left\langle n_{H}^{2}\right\rangle /\left\langle n_{H}\right\rangle^{2}$ declines very sharply at high redshift, and is $C \approx 2$ at $z=20$, compared to $C \approx 30$ at $z=10$ (Haiman, Abel \& Madau 2001). Hence, the recombination time $t_{\mathrm{rec}}=1 / \alpha_{B} n_{e} C$ is in fact longer by a factor $\sim(30 / 2) /(20 / 10)^{3} \sim 2$ at $z=20$. If (many generations of) VMSs form over a Hubble time $t_{\mathrm{H}}$ at $z \sim 20$, then the number of recombinations is $n_{\text {rec }} \sim$ $t_{\mathrm{H}} / t_{\mathrm{rec}} \approx(9,50)[(1+z) / 20]^{1.5}(C / 2)$ per HII and HeIII particle, respectively. Since the halo star abundances suggest that $n_{\text {ion }} \sim(3-7,30-70,8-15)$ photons above the (HI,HeI,HeII) ionization threshold per $\mathrm{H}$ or $\mathrm{He} n u-$ cleus were produced, the filling factor of HII and HeIII regions due to VMSs may be $\sim n_{\text {ion }} / n_{\text {rec }} \sim 0.35-0.75$ and $\sim 0.15-0.30$ respectively. We may have underestimated the ionizing photon production rate and thus the filling factors by factors of a few, since we do not count VMSs which collapse to black holes and do not eject their metals. It is thus plausible that the IGM was completely ionized by VMSs. Once the IGM is reionized, a much lower comoving emissivity is required to keep it ionized at lower redshifts than to ionize it $a b$ initio: the high IGM temperature strongly suppresses gas accretion onto low mass halos $v_{c}<30 \mathrm{~km} \mathrm{~s}^{-1}$, so the gas clumping factor remains small at lower redshift. This could reduce the comoving emissivity required for an ionized IGM by a factor $C \sim 30$ at $z=10$.

Our estimate that $f_{\mathrm{VMS}} \sim 3-7 \times 10^{-5}$ is consistent with other estimates. Due to feedback processes, it is unlikely that more than one VMS forms per halo (Madau \& Rees 2001). If VMSs form in the first pregalactic objects able to cool within a Hubble time, with masses $\mathrm{M}>\mathrm{M}_{\text {crit }}=4 \times 10^{5}\left(\frac{1+\mathrm{z}}{25}\right)^{-2} \mathrm{M}_{\odot}$, at a time when such halos collapse from $2.5 \sigma$ density peaks in the universe and contain $\sim 1.3 \%$ of the total mass of the universe, then $f_{\mathrm{VMS}} \sim 1.3 \times 10^{-2} \times M_{\mathrm{VMS}} /\left(f_{B} M_{\text {crit }}\right)=5 \times 10^{-5}$ (where $\left.f_{B} \equiv \Omega_{b} / \Omega_{m}\right)$. Normal star formation probably starts when the gas metallicity is sufficiently high for efficient metal-line cooling, reducing the Jeans mass and enhancing fragmentation. Bromm et al. (2001b) find from numerical simulations that this is likely to take place once $Z$ reaches 1 to $10 \times 10^{-4} Z_{\odot} \sim 2$ to $20 \times 10^{-6}$. Similarly, Hellsten \& Lin (1997) find that the critical metallicity for isobarically cooling gas at $n T \sim 10^{4} \mathrm{~K} \mathrm{~cm}^{-3}$ to cool to $T \sim 50 \mathrm{~K}$ is $[\mathrm{Fe} / \mathrm{H}] \sim-3$, the critical metallicity identified by WQ. Since half the main-sequence mass of a VMS becomes metals in the HW models, our estimate of $f_{V M S}$ corresponds roughly to $Z \sim 0.5 f_{V M S} \sim 1.5$ to $3.5 \times 10^{-5}$. However, caution is necessary because the cooling studies used solar metal abundances scaled with metallicity, which are very different from the HW yields. The difference may be unimportant if oxygen is the dominant coolant (since $\mathrm{O}$ yields from $\mathrm{HW}$ scale with metallicity in roughly solar ratios, see Fig. 1), but a direct comparison requires a rigorous calculation of the cooling properties of gases with non-solar ratios of metal abundances.

Finally, due to the high effective temperature of VMSs, HeII may reionize at high redshift in VMS reionization scenarios, only to recombine once ordinary stars with softer spectra become predominant (subsequent HeII reionization only took place at $z \sim 3$, as observed by Kriss et al 2001). In standard stellar reionization scenarios, HeII reionization takes place only once, when quasars turn on. The higher temperatures achievable with early HeII reionization would have interesting consequences for the thermal history of the IGM (Miralda-Escude \& Rees 1994; Abel \& Haehnelt 1999), and the HeII recombination lines produced in the host halo may eventually be observable with NGST (Oh, Haiman \& Rees 2001; Tumlinson, Giroux \& Shull 2001).

We thank A. Heger for providing his calculations of the metal yields from very massive stars. Support for this work was provided by NSF grant AST-0096023 (S.P.O.), by NASA through ATP grant NAG5-4236 (P.M.), and by NASA NAG5-10293 and Caltech Division Contribution 8771(1082) (K.M.N. \& G.J.W.).

\section{REFERENCES}

Abel, T. \& Haehnelt, M. G. 1999, Ap.J, ApJ, 520, L13

Abel, T., Bryan, G.L., \& Norman, M.L., 2000, ApJ, 540, 39

Baraffe, I., Heger, A., \& Woosley, S.E., 2001, ApJ, 550, 890 (HW)

Bromm V Coppi P.S., \& Larson, R.B., 2001a, ApJ, submitted, astro-ph/0102503

Bromm, V., Ferrara, A Coppi,P.S., \& Larson, R.B. 2001b, MNRAS, submitted, astro-ph/0104271

Bromm, V., Kudritzk1, R.P., \& Loeb, A. 2001c, ApJ, 552, 464

Carr, B.J., Bond, J.R., \& Arnett, W.D. 1984, ApJ, 277, 445

Carretta, E., Gratton, R., \& Sneden, C. 2000, A\&A, 356, 238

Cowie, L.L., \& Songaila, A. 1998, Nature, 394, 248

Ezer, D. \& Cameron, A.G.W., 1971, Ap\&SS, 14, 399

Ellison S.I, Songaila, A, Schaye, I, Pottini, M, 2000, AI, 120,1175

Fuller, T.M., \& Couchman, H.M.P. 2000, ApJ, 544, 6

Fryer, C., Woosley, S., \& Heger, A. 2001, ApJ, 550, 372

Gnedin, N.Y., \& Ostriker, J.P. 1997, ApJ, 486, 581

Haiman, Z., Thoul, A.A., \& Loeb, A., 1996, ApJ, 464, 523

Haiman, Z., \& Loeb, A., 1997, ApJ, 483, 21

Haiman, Z., Abel, T., \& Madau, P. 2001, ApJ, 55 550

Heger, A., \& Woosley, S.E., 2001, submitted, fotro-ph/0107037

Hellsten, U., \& Lin, D.N.C. 1997, astro-ph/9708086

Israelian, G., Rebolo, R., Garcia Lopez, K.J., Bonıtacio, R., Molaro, R., Basrio, G., Shchikina, N. 2001, ApJ, 551, 833

Kriss, G.A., et al, 2001, Science, 293, 1112

Kudritzki, R.P, 2000, in The First Stars, Weiss, A., Abel, T., Hill, V. (eds), Springer, Berlin, p. 127
Larson, R.B. 1998, MNRAS, 301, 569

Madau, P., Ferrara, A., \& Rees, M.J. 2001, ApJ, 554, 92

Madau, P., \& Rees, M.J. 2001, ApJ, 551, L27

Makino, N. Sasaki, S., \& Suto, Y. 1998, ApJ, 497, 555

McWilliam, A., Preston, G.W., Sneden, C., \& Searle, L. 1995, AJ, 109, 2757

Miralda-Escudé, J., \& Rees, M. J. 1994, MNRAS, 266, 343

Miralda-Escudé, J., \& Rees, M. J. 1997, ApJ, 487, 21

Nakamura, F., \& Umemura, M. 2001, ApJ, 548, 19

Oh, S.P., Haiman, Z., \& Rees, M.J. 2001, ApJ, 553, 730

Portinari, L., Chiosi, C., Bressan, A. 1998, A\&A, 334, 505

Qian, Y.-Z., \& Wasserburg, G.J. 2001a, ApJ, 549, 337

Qian, $Y . Z$ \& Wasserburg, G.J. 2001b, ApJ, in press, astro$\mathrm{ph} / 0106085$

Ryan, S.G. 2000, in "The Galactic Halo: From Globular Clusters to Field Stars", ed. A. Noels, P. Magain, D. Caro, E. Jehin, G. Parmentier, \& A. Thoul, p. 101

Schaye, J., Rauch, M., Sargent, W.L. W., Kim, T.-S., 2000, ApJ, 541, L1

Schwarzschild, M., \& Spitzer, L. 1953, The Observatory, 73, 77

Tumlinson, J., Giroux, M.L., Shull, M.J. 2001, ApJ, 550, L1

Tumlinson, J., \& Shull, M.J. 2000, ApJ, 528, L65

Wasserburg, G.J., Busso, M., \& Gallino, R. 1996, ApJ, 466, L109

Wasserburg, G.J., \& Qian, Y.-Z. 2000, ApJ, 539, L99 